\title{
Telomere shortening in smokers with and without COPD
}

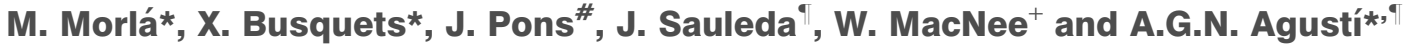

\begin{abstract}
Telomeres are complex DNA-protein structures located at the end of eukaryotic chromosomes. Telomere length shortens with age in all replicating somatic cells. It has been shown that tobacco smoking enhances telomere shortening in circulating lymphocytes. The present study investigated whether this effect was further amplified in smokers who develop chronic obstructive pulmonary disease.
\end{abstract}

Telomere length was determined by fluorescence in situ hybridisation in circulating lymphocytes harvested from 26 never-smokers, 24 smokers with normal lung function and 26 smokers with moderate-to-severe airflow obstruction (forced expiratory flow in one second $48 \pm 4 \%$ predicted).

In contrast to never-smokers, telomere length significantly decreased with age in smokers. There was also a dose-effect relationship between the cumulative long-life exposure to tobacco smoking (pack-yrs) and telomere length. The presence and/or severity of chronic airflow obstruction did not modify this relationship.

The results of the current study confirm that smoking exposure enhances telomere shortening in circulating lymphocytes. It also demonstrates a dose-effect relationship between exposure to tobacco smoking and telomere length, but failed to show that this effect is amplified in smokers who develop chronic obstructive pulmonary disease.

KEYWORDS: Ageing, chronic bronchitis, emphysema, inflammation, repair, tobacco

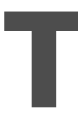
obacco smoking is the main risk factor for chronic obstructive pulmonary disease (COPD) [1]. However, for reasons that are still poorly understood, only a percentage of smokers develop the disease [1].

Very recently, VALDES et al. [2] have shown that tobacco smoking enhances telomere loss in circulating lymphocytes. Telomeres are complex DNA-protein structures located at the end of eukaryotic chromosomes, which shorten with age in all replicating somatic cells [3, 4]. Telomere length is, therefore, a marker of cell ageing and senescence [5].

An accelerated rate of lung function decline with age is one of the central pathophysiological characteristics of COPD [1]. In the present study, the authors hypothesised that the effects of tobacco smoking upon telomere length shortening would be enhanced in smokers who developed COPD, compared with those whose lung function was preserved despite their habit. To test this hypothesis, the rate of telomere shortening was compared with age in circulating lymphocytes harvested from smokers with and without COPD.

\section{METHODS}

\section{Population and ethics}

The study included 50 life-long smokers, 24 of whom had normal lung function and 26 with evidence of COPD according to international guidelines [1], and 26 never-smokers. Participants with chronic diseases other than COPD were excluded. The Ethics Committee of Hospital Universitari Son Dureta (Palma De Mallorca, Spain) approved the study and patients gave their informed consent.

\section{Lung function measurements}

Forced spirometry was obtained in all participants according to international recommendations [6]. Reference values were those of a Mediterranean population [7].

\section{Lymphocyte isolation}

Peripheral blood samples were obtained by venipuncture. Lymphocytes were isolated by mixing $10 \mathrm{~mL}$ of venous blood with an equal volume of PBS. This was followed by centrifugation on a 2-mL layer of a Ficoll-Paque research grade gradient (Pharmacia Biotech, Uppsala, Sweden) at $900 \times g$ for 30 minutes at $22^{\circ} \mathrm{C}$. The
AFFILIATIONS

*Unitat d'Investigació

\#Serveis de Inmunologia, and

"Pneumologia, Hospital Universitari Son Dureta, Palma de Mallorca, Spain.

+ELEGI Laboratories, Royal Infirmary, University of Edinburgh, Scotland, UK.

CORRESPONDENCE

$X$. Busquets

IUNICS-Hospital Universitari Son Dureta

Andrea Doria 55

07014- Palma de Mallorca

Spain

Fax: 34971175228

E-mail: busquets@hsd.es

Received:

July 252005

Accepted after revision:

November 182005

\section{SUPPORT STATEMENT}

Supported, in part, by Fondo de Investigaciones Sanitarias (Red Respira RTIC 03/11 and FIS 020978), Sociedad Española de Pneumología y Cirugía Torácica (SEPAR), Govern Balear and Associacio Balear per l'Estudi de Malalties Respiratories (ABEMAR) 
lymphocyte band was removed, washed once with PBS and resuspended with telomere probe hybridisation solution (Telomere PNA Kit/FITC for Flow Cytometry; Dako, Glostrup, Denmark).

\section{Measurement of telomere length}

The present authors used in situ hybridisation of a fluoresceinconjugated peptide nucleic acid (PNA) telomere probe/ fluorescein isothiocyanate (FITC) [4] to lymphocytes in solution, following the directions of the manufacturer (Telomere PNA Kit/FITC for Flow Cytometry; Dako). Telomere length was determined as relative telomere length (RTL) by comparison with an internal cell line control (1301 cells) [4]. This cell line is characterised by a lack of telomere shortening (and hence, constant telomere length) during cell division due to overexpression of telomerase [4]. Briefly, a suspension containing a mixture of $2 \times 10^{6}$ lymphocytes and $2 \times 10^{6}$ of 1301 cells was denatured at $82^{\circ} \mathrm{C}$ for $10 \mathrm{~min}$, with and without the fluorescein-conjugated PNA telomere probe in the hybridisation solution. Samples were hybridised overnight at room temperature and washed at $40^{\circ} \mathrm{C}$ with washing solution. They were then re-suspended with PBS, containing DNA-staining solution buffer. Hybridisation, washing and DNA-staining solutions were provided by the manufacturer (Dako). The correct hybridisation of the probe was confirmed by fluorescence microscopy and Southern blot analysis (fig. 1). Quantification of RTL was carried out by flow fluorescence in situ hybridisation (FISH) analysis in an EPICS XL Flow Cytometer (Beckman Coulter, Fullerton, CA, USA), by comparing telomere length in sample cells with that of 1301 cells.
Figure 2 shows a representative example of RTL determination in a 61-yr-old nonsmoking donor and 1301 cells $(100 \%$ telomere length). As shown in figure $2 \mathrm{a}$, cells were gated on region 1 (R1) on the basis of propidium iodide (PI) fluorescence and forward light scatter. Regions 2 (donor lymphocytes) and 3 (1301 cell; R2 and R3) were selected within R1 from forward versus side scatter dot plot histograms (fig. $2 \mathrm{~b}$ ). Figures $2 \mathrm{c}$ and d show forward versus side scatter dot plot histograms of R2 and R3 with (d) and without (c) FITC-labelled PNA telomere probe. Telomere fluorescence was calculated by subtracting the mean background fluorescence from the mean fluorescence obtained with the telomere probe, with (shaded histograms) or without (open histograms) FITC-labelled PNA telomere probe (e and $\mathrm{f}$ ).

\section{Statistical analysis}

Results are expressed as mean \pm SEM. The unpaired t-test was used to compare groups. Pearson correlation analysis was used to investigate potential relationships between variables of interest.

\section{RESULTS}

Table 1 shows the main clinical and functional characteristics of the population studied. All participants were males of similar age. Cumulative smoking exposure (pack-yrs) was not significantly different between smokers with normal lung function and COPD patients. By definition, spirometry was normal in never-smokers and in smokers with normal lung function, whereas patients with COPD showed moderate-tosevere airflow obstruction [1].
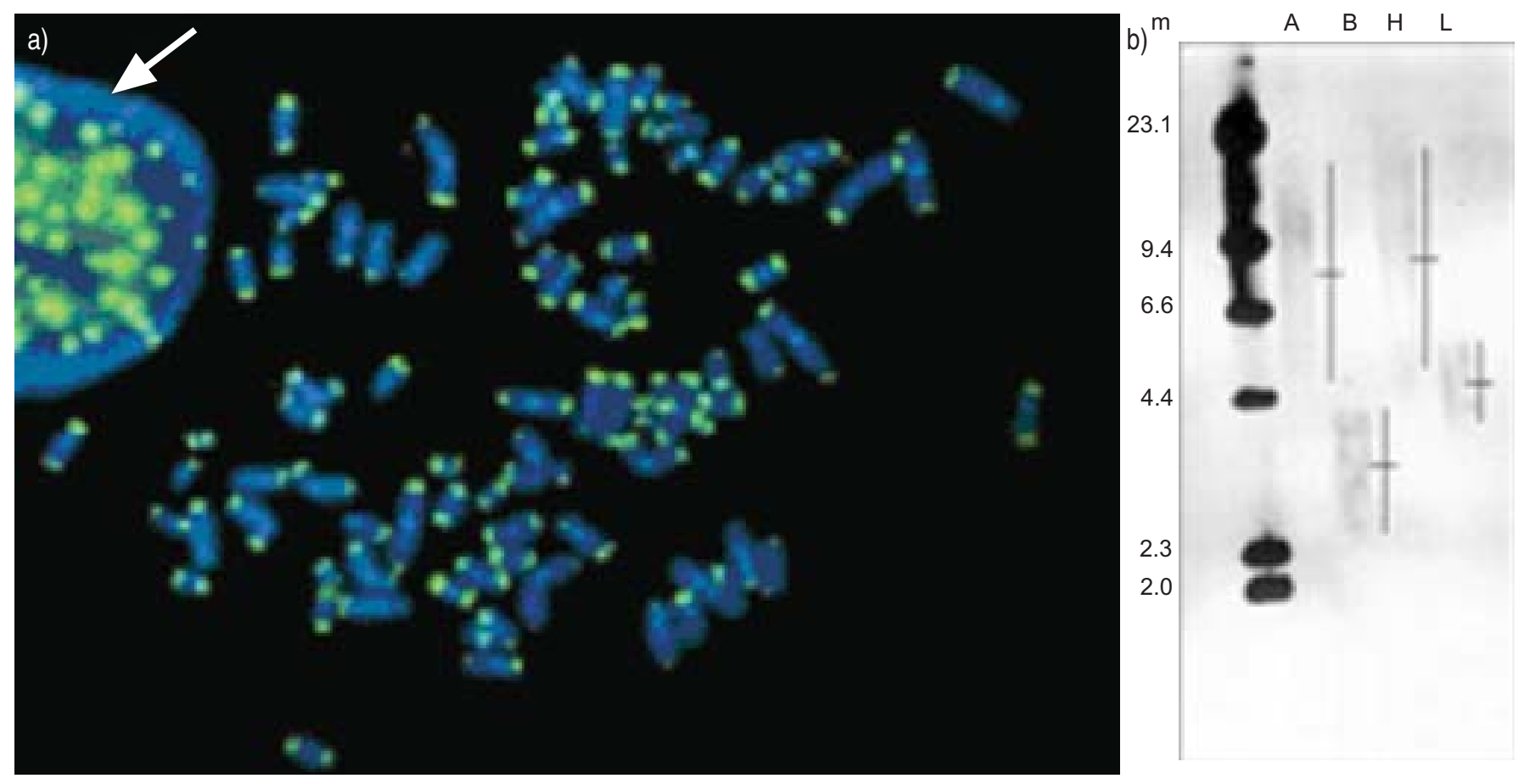

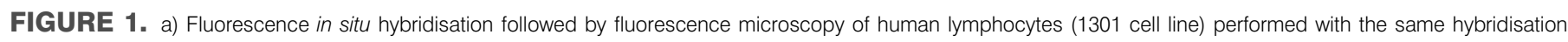

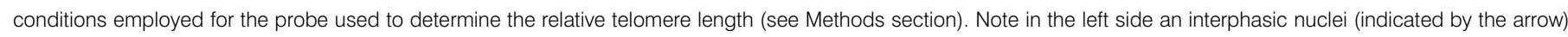

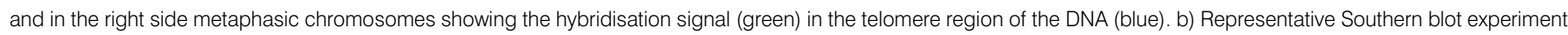

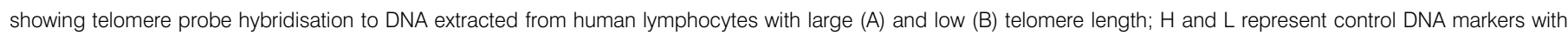
large and low telomere length, respectively. 

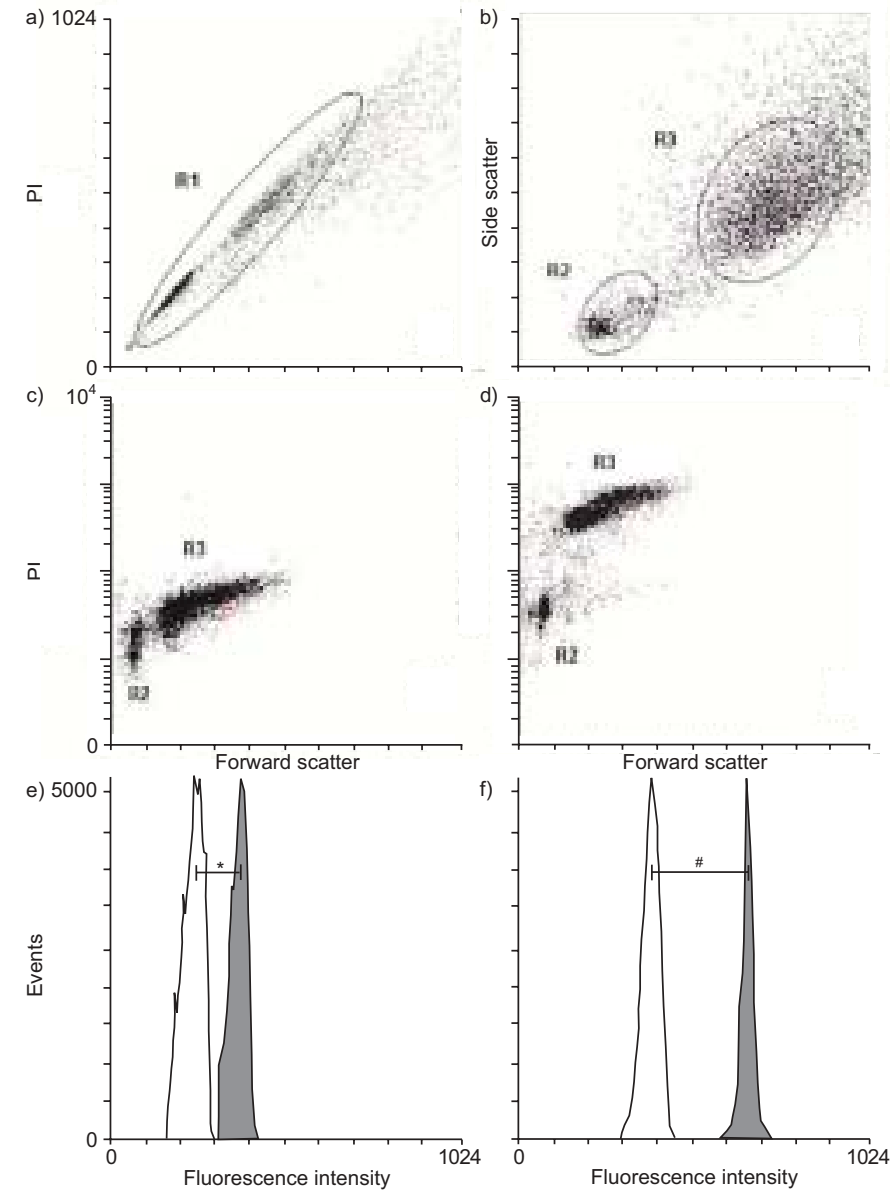

FIGURE 2. a-d) Flow fluorescence in situ hybridisation analysis of periphera lymphocytes from a healthy 61-yr-old non smoker donor and 1301 human lymphocyte tetraploid cells (internal reference value: 100\% telomere length) analysed after hybridisation with $(\square)$ and without $(\square)$ fluorescein isothiocyanatelabelled peptide nucleic acid telomere probe (e and f). e) Refers to region 2 (R2) and f) refers to region 3 (R3). See the Methods section for details. In this particular example (e and f), relative telomere length (RTL) was calculated as (mean FL1 sample cells with probe-mean FL1 sample cells without probe) $\times$ DNA index of control cells $\times 100 /$ (mean FL1 1,301 cells with probe-mean FL1 control cells without probe) $\times$ DNA index of sample cells. Hence, RTL $=(34.4-15.1) \times 2 \times 100 /(384.8-$ 29.3) $\times 1=19.3 \times 2 \times 100 / 355.5 \times 1=10.85 \%$. FL1: fluorescence detector $1 ; \mathrm{Pl}$ : propidium iodide; R1: region 1. *: 19.3; *: 355.5 .

As shown in figure 3a, within the age range studied RTL did not decrease with increasing age in never-smokers. In contrast, within the same age range, RTL decreased significantly with age in smokers (fig. 3b), irrespective of the presence (closed symbols) or absence (open symbols) of COPD. The influence of cumulative smoking exposure on RTL is further supported by the significant dose-effect relationship found between packyrs and RTL (fig. 3c). The overlap between smokers with normal lung function (open symbols) and COPD (closed symbols) suggests that this effect is independent of the presence or absence of chronic airflow obstruction.

\section{DISCUSSION}

The present study confirms the recent data published by VALDES et al. [2] and shows that tobacco smoking causes

\begin{tabular}{|c|c|c|c|}
\hline \multirow[t]{2}{*}{ TABLE 1} & \multicolumn{3}{|c|}{$\begin{array}{l}\text { Slinical and functional characteristics of the } \\
\text { opulation studied }\end{array}$} \\
\hline & Vever-smokers & $\begin{array}{l}\text { Smokers with } \\
\text { normal lung function }\end{array}$ & $\begin{array}{l}\text { Smokers with } \\
\text { COPD }\end{array}$ \\
\hline Subjects $n$ & 26 & 24 & 26 \\
\hline Age yrs & $57 \pm 2$ & $58 \pm 2$ & $61 \pm 1$ \\
\hline Sex male/female & $26 / 0$ & $24 / 0$ & $26 / 0$ \\
\hline $\begin{array}{l}\text { Smoking history } \\
\text { pack-yrs }\end{array}$ & $0 \pm 0$ & $39 \pm 4^{\#}$ & $49 \pm 3^{\#}$ \\
\hline FEV $1 \%$ reference & $106 \pm 3$ & $94 \pm 2^{\#}$ & $48 \pm 4^{\#, \cdot}$ \\
\hline FEV $1 /$ FVC $\%$ & $80 \pm 1$ & $82 \pm 3$ & $52 \pm 2^{\# \cdot \cdot}$ \\
\hline
\end{tabular}

enhanced shortening of telomere length (figs $3 a$ and $b$ ). However, it expands on that study's results because: 1) it shows a dose-response relationship between cumulative exposure to tobacco smoking and telomere length shortening in circulating lymphocytes (fig. 3c); and 2) for the first time, the potential role of smoking-induced telomere shortening is considered in the pathogenesis of COPD. Unfortunately, the present study could not demonstrate a differential effect of smoking on telomere length according to the presence or absence of COPD (figs $3 b$ and c).

Several potential mechanisms can help explain these observations. First, tobacco smoking is known to be associated with low-grade systemic inflammation [8], particularly in patients with COPD [9]. Systemic inflammation may cause increased lymphocyte turnover and a shift from naïve (CD45RA+) to memory (CD45RO+) T-cells. The latter are known to have shorter telomeres [4] and may therefore help explain the observations of this study. To address this possibility, the authors compared the percentage of CD45RA+ and CD45RO+ in smokers and never-smokers; no significant difference was detected (data not shown). This argues against increased lymphocyte maturation due to chronic inflammation as an explanation for the enhanced loss of telomere length observed in smokers. However, tobacco smoking is a well-known cause of systemic oxidative stress [10], and progressive oxidative damage has been implicated in ageing and age-related pathologies [11]. It is likely, therefore, that the accelerated loss of telomere length observed in the present study in smokers is part of an oxidantinduced senescence phenomenon [2]. If this is the case, this may have important pathogenic implications because cell senescence jeopardises the capacity to repair tissue injury [12].

The present study has some limitations that deserve comment. First, no reduction in telomere length was seen in relation to increasing age in never-smokers (fig. 3a). Previous studies in the general population have reported that telomere length in circulating lymphocytes shortens physiologically (albeit moderately) from 0 to $90 \mathrm{yrs}$ of age [4]. The relatively narrow age range of the present study (40-75 yrs) and its smaller sample size (in comparison with previous studies in the 

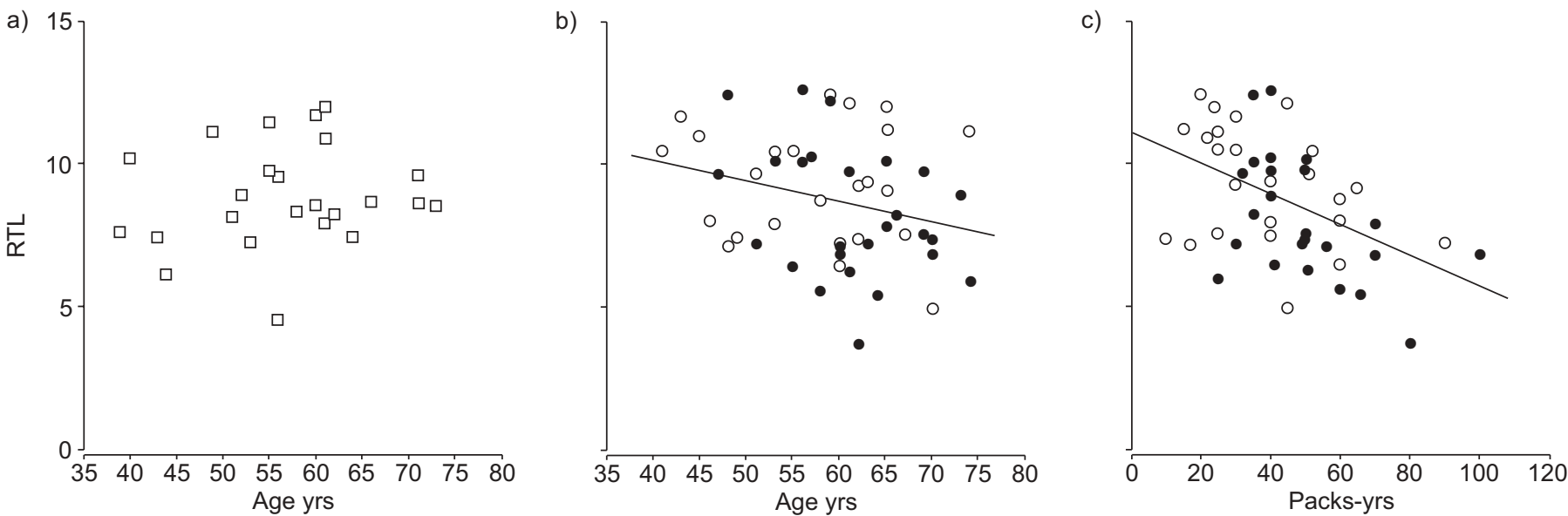

FIGURE 3. Relationship between relative telomere length (RTL) and age, both in a) nonsmokers ( $p$-value is nonsignificant) and $b)$ smokers ( $p=0.05, r=-0.27)$. $c$ ) Cumulative smoking exposure (pack-yrs; $p<0.001, r=-0.45$ ). $\square$ : never smokers; $\bigcirc$ : smokers with normal lung function;

smokers with chronic obstructive pulmonary disease.

general population [4]) may have limited the ability to detect a small change of telomere length in never-smokers. However, previous studies have not excluded smokers [4, 13], so it is possible that part of the shortening in telomere length observed in these previous studies could in fact be attributed to smoking and not exclusively to ageing. Whatever the case, the present results confirm that tobacco smoking accelerates telomere length shortening in circulating lymphocytes [2].

Secondly, the sample size of the present study (50 life-long smokers and 26 controls of normal lung function) is relatively small, and is in the range of a previous work that studied the relationship between telomere length and atherosclerosis, another very common age- and smoking-related condition [13]. In this study, SAMANI et al. [13] described shorter telomere length in the circulating lymphocytes of 10 patients with atherosclerosis, compared with 20 appropriate controls. Considering the observed mean RTL values in smokers and nonsmokers (as well as the observed standard deviation) and assuming an alpha error of 0.05 and a beta error of 0.1 (bilateral), the present study would need to include $\sim 400$ individuals in each group (1,200 in total) which is much beyond the capacity of this paper. Future studies should confirm this pilot study by investigating these effects in larger populations and in lung tissue, since these observations open up a previously unexplored pathogenic avenue in COPD, particularly in relation to the capacity of the lung to repair smoking-induced tissue injury [12].

In summary, the present study paper confirms the findings of previous studies [2], showing that tobacco smoking is significantly associated with telomere loss in circulating lymphocytes. It has been shown that this occurs in a doseresponse manner. However, the study failed to show that this phenomenon is enhanced in smokers who develop chronic obstructive pulmonary disease.

\section{REFERENCES}

1 Celli BR, MacNee W, Agusti AG, et al. Standards for the diagnosis and treatment of patients with COPD: a summary of the ATS/ERS position paper. Eur Respir J 2004; 23: 932-946.

2 Valdes AM, Andrew T, Gardner JP, et al. Obesity, cigarette smoking, and telomere length in women. Lancet 2005; 366: 662-664.

3 Frenck RW Jr, Blackburn EH, Shannon KM. The rate of telomere sequence loss in human leukocytes varies with age. Proc Natl Acad Sci USA 1998; 95: 5607-5610.

4 Rufer N, Brummendorf TH, Kolvraa S, et al. Telomere fluorescence measurements in granulocytes and T lymphocyte subsets point to a high turnover of hematopoietic stem cells and memory $\mathrm{T}$ cells in early childhood. J Exp Med 1999; 190: 157-167.

5 Epel ES, Blackburn EH, Lin J, et al. Accelerated telomere shortening in response to life stress. Proc Natl Acad Sci USA 2004; 101: 17312-17315.

6 American Thoracic Society Official Statement. Standardization of spirometry. 1994 update. Am J Respir Crit Care Med 1995; 152: 1107-1136.

7 Roca J, Sanchis J, Agustí-Vidal A, et al. Spirometric reference values for a mediterranean population. Bull Eur Physiopathol Respir 1986; 22: 217-224.

8 van Eeden SF, Yeung AC, Quinlam K, Hogg JC. Systemic response to ambient particulate matter. Relevance to chronic obstructive pulmonary disease. Proc Am Thorac Soc 2005; 2: 61-67.

9 Agusti AG, Noguera A, Sauleda J, Sala E, Pons J, Busquets $X$. Systemic effects of chronic obstructive pulmonary disease. Eur Respir J 2003; 21: 347-360.

10 van der Vaart H, Postma DS, Timens W, ten Hacken NH. Acute effects of cigarette smoke on inflammation and oxidative stress: a review. Thorax 2004; 59: 713-721.

11 Finkel T, Holbrook NJ. Oxidants, oxidative stress and the biology of ageing. Nature 2000; 408: 239-247.

12 Toussaint O, Medrano EE, von Zglinicki T. Cellular and molecular mechanisms of stress-induced premature senescence (SIPS) of human diploid fibroblasts and melanocytes. Exp Gerontol 2000; 35: 927-945.

13 Samani NJ, Boultby R, Butler R, Thompson JR, Goodall AH. Telomere shortening in atherosclerosis. Lancet 2001; 358: 472-473. 